\title{
¿LIBERTADES A CAMBIO DE SEGURIDAD?
}

\section{¿LIBERTIES IN EXCHANGE FOR SAFETY?}

\section{AUTORES}

Jesus Cea Avion: Ingeniero Técnico Superior de Telecomunicaciones. Director Técnico e I+D en HispaSec (España)

jcea@argo.es

\section{CURRÍCULUM VITAE}

Ingeniero Técnico Superior de Telecomunicaciones. Director Técnico e I+D en HispaSec, empresa especializada en seguridad informática, consultoría, auditoría y desarrollo. Interesado tanto en los aspectos criptográficos como en las implicaciones sociales y legales del uso de tecnología criptográfica. Miembro de Fronteras Electrónicas España www.arnal.es/free. Más información sobre criptología en la página www.jcea.es/cripto.htm y artículos en www.jcea.es/artic.

\section{RESUMEN}

Desde hace años existe un debate en EE.UU. sobre el uso privado de la criptografía para asegurar la confidencialidad de las comunicaciones entre particulares. El argumento de los que están en contra es que si una comunicación está cifrada, las fuerzas del orden no pueden espiarlas para detectar actividades criminales. Proponen o bien prohibir la criptografía por ley, o dotar a los programas criptográficos de Puertas Traseras. 


\section{PALABRAS CLAVE}

Libertades - Seguridad - Criptografía - Atentados

\section{ABSTRACT}

For years there is a debate in the U.S. on the private use of cryptography to ensure confidentiality of communications between individuals. The argument of those against is that if a communication is encrypted, the security forces can not spy on them to identify criminal activity. Proposed ban cryptography or by law, or provide cryptographic programs Backdoors.

\section{KEY WORDS}

Freedoms - Security - Cryptography - Attacks

Los atentados recientes en EE.UU. abonan el campo para nuevas leyes que pretenden proporcionar más seguridad a los ciudadanos a costa de un mayor control gubernamental.

A continuación se adjunta un mensaje que escribí hace unas semanas para un foro del que formo parte pero que plantea una evolución previsible en el uso y reconocimiento de libertades civiles, sobre todo en lo que se refiere al binomio 
libertad/seguridad. El mensaje se incluye tal y como fue enviado, sin ningún tipo de edición.

Subject: [Nosotros] Debate: ¿libertades a cambio de seguridad?

Date: Tue, 25 Sep 2001 12:32:29 +0200

From: Jesus Cea Avion <jcea@argo.es>

Me gustaría proponer un debate a los contertulios, relativo a los movimientos que se empiezan a oler en EE.UU. tendentes a limitar "algo" las libertades a cambio de una "presunta" mejora en la seguridad.

Elaboraré más mi postura si es necesario a lo largo del debate, si lo hay, pero mi posición personal es que no se puede comerciar con las libertades, y menos para obtener una seguridad adicional más que dudosa. Las libertades que se pierden tardan mucho en recuperarse, o no se recuperan nunca.

Lo peor de todo es que, encima, no veo ninguna razón incontestable para que esa pérdida de libertad incremente la seguridad.

Un ejemplo:

Desde hace años existe un debate en EE.UU. sobre el uso privado de la criptografía para asegurar la confidencialidad de las comunicaciones entre particulares. El argumento de los que están en contra es que si una comunicación está cifrada, las fuerzas del orden no pueden espiarlas para detectar actividades criminales. Proponen o bien prohibir la criptografía por ley, o dotar a los programas criptográficos de "puertas traseras". 
Para cualquier persona "normal y corriente", este argumento tiene su lógica y parece razonable.

Pero no lo es. No lo es por tres razones:

* La gente que usa la criptografía con fines delictivos son delincuentes y, por definición, no seguirán las leyes. Si no existen programas criptográficos, crearán los suyos propios (es muy fácil y existen infinidad de algoritmos de alta calidad documentados en cualquier libro o revista sobre la materia), sin puertas traseras.

* Si el usuario doméstico no puede usar la criptografía, mientras que el delincuente lo hará de todas formas, la seguridad de sus comunicaciones decrecerá. El que nada tiene que ocultar estará a merced del "gran hermano", de organizaciones mafiosas, de terroristas o del trabajador malicioso de su ISP. Esa gente, que no tiene nada que ocultar, estará completamente desprotegida.

Yo no tengo nada que ocultar, pero las cartas que envío a mi madre las mando en un sobre cerrado. ¿Mañana se me obligará a enviar todo en forma de postal, abierto, para que la policía pueda comprobar que no envío los planos de una bomba atómica?. Por cierto, cualquiera que sepa algo de física puede construirse una... si cuenta con los materiales apropiados; no hace falta que nadie le mande los planos.

* Si un programa tiene puertas traseras, ¿Quien controla su uso correcto?. ¿Quien controla que mañana, por ejemplo, un grupo mafioso o terrorista consiga el acceso a dicha puerta?. Sería peor que no tener criptografía, porque piensas que estás seguro cuando no es así.

La criptografía es una tecnología y, como algunos estamos cansados de repetir, la tecnología es neutral. Se puede usar tanto para lo bueno como para lo malo. La 
gasolina puede provocar explosiones, incendios y contamina cosa mala, pero no la vamos a prohibir a corto plazo. Los cuchillos provocan numerosos accidentes y son armas utilizadas en la mayoría de los delitos de sangre, y tampoco los vamos a prohibir. ¿Con qué cortaría yo el jamón si no?.

Otro ejemplo evidente es el de la biometría. Se quiere, por ejemplo, desplegar sistemas de reconocimiento facial, escáneres de retina o de huella dactilar a la entrada de un estadio de fútbol, por ejemplo. El objetivo es detectar al momento elementos terroristas y criminales buscados por la policía.

Todo muy bueno y muy bonito.

Por supuesto, en cuanto los criminales sepan que tendrán que pasar por un sistema de ese tipo, sencillamente verán el fútbol desde su casa. El terrorista que quiere poner una bomba se colará por la salida de incendios o sobornará a un guardia... o no estará fichado.

Pero, ¿y el usuario "particular"?

Un día te encontrarás que, cuando llevas a tu hijo al partido, la seguridad del estadio te retiene por tener una multa pendiente o por no haber devuelto tu último libro a la biblioteca. Asimismo, un día te empezará a llegar publicidad sobre botas de fútbol, porque "alguien" ha filtrado que eres un habitual del estadio.

Peor aún, pasado mañana tu compañía aseguradora te subirá las cuotas de tu seguro de vida porque se ha demostrado que asistir a un partido de fútbol incrementa las probabilidades de sufrir un infarto. 
La semana que viene te encontrarás tu coche destrozado porque "alguien" lo ha golpeado con un bate de beisbol. Tu nunca sabrás la razón, pero un hincha del equipo rival del pasado domingo, que perdió en tu campo, vive en tu misma calle y "sabe mucho de internet".

Un tercer punto: se está abogando por incrementar la monitorización de Internet, meter el famoso "carnívoro" para escanear mensajes buscando "cosas inconvenientes", etc. El argumento es que los terroristas usan internet para coordinarse.

Dios, ¡menudo sinsentido!.

Cuando ETA envía un paquete bomba a un periodista, en España, nadie se plantea denunciar a correos y obligarle a analizar todos y cada uno de los paquetes que gestiona. La mayoría de los crímenes se planean por teléfono (no por Internet), y nadie se ha planteado el pinchar absolutamente todas las llamadas sin orden judicial.

¿Por qué Internet es tan diferente?. ¿Por qué ese agravio comparativo?.

En EE.UU., por una vez, ya no son sólo las asociaciones pro derechos civiles las que están preocupadas, sino que muchos ciudadanos (y, afortunadamente, medios de comunicación), se están dando cuenta de que las propuestas de ley que se están debatiendo en la actualidad en EE.UU. son lobos en piel de cordero, y que sus implicaciones a medio y largo plazo son aterradoras.

Las leyes antiterroristas que se plantean, de aprobarse su borrador actual, convertirán EE.UU. en el mayor estado policial que la humanidad haya conocido nunca. 
Recomiendo a todos los lectores de "Una Al Día" que lean con atención los documentos que siguen. Considerando el colonialismo cultural de EE.UU. sobre el mundo occidental, habrá que seguir muy de cerca la evolución legislativa en EE.UU., en previsión de una posible exportación a Europa en un futuro más o menos inmediato.

La mayoría de los enlaces han sido recopilados y difundidos por RRE (Red Rock Eater News Service). Nuestro agradecimiento.

Opina sobre esta noticia:

http:/ / www.hispasec.com/ unaaldiacom.asp?id=1080

Más información:

Red Rock Eater News Service

http://dlis.gseis.ucla.edu/people/pagre/rre.html

House Bill Would Expand Federal Detention Powers

http:/ / www.washingtonpost.com/wp-dyn/articles/ A55410-2001Oct1.html

Defining Terrorism Stirs Words of Dispute

http:/ / www.latimes.com/templates/misc/printstory.jsp?slug=la\%2D100101legal

Statement on Terrorism, Civil Liberties, and the Internet

http://www.pfir.org/statements/liberties

Don't Blame the Internet

http:/ / www.washingtonpost.com/wp-dyn/articles/ A43828-2001Sep29.html

analysis of the anti-hacking provisions of the proposed anti-terrorism act

http:/ / www.politechbot.com/p-02597.html

British ID Cards Backlash Begins

http://www.guardian.co.uk/humanrights/story/0,7369,561043,00.html 
"Amazing" Lapse in Security Cited at Logan

http://www.boston.com/dailyglobe2/274/metro/_Amazing_lapse_in_security_cite d_at_Logan+.shtml

Will Other Voices Be Heard?

http://chicagotribune.com/features/lifestyle/chi-0110010037oct01.story

the very dangerous attacks on Bill Maher

http://www.dailyhowler.com/h100101_1.shtml

Censorship and the War on Terrorism

http://www.mediachannel.org/views/interviews/macarthur.shtml

We Must Dismantle Our Democracy in Order to Save It

http:/ / www.salon.com/comics/tomo/2001/10/01/tomo/

Muslim Leaders Struggle With Mixed Messages

http://www.washingtonpost.com/wp-dyn/articles/ A55677-2001Oct1.html

the followers of John Adams are bringing back the Alien and Sedition Acts

http://www.salon.com/politics/feature/2001/10/03/ashcroft/print.html

conservative dupes learn what they really voted for

http:/ / www.nytimes.com/2001/10/03/national/03WEST.html

Anti-Terrorism Bill Hits Snag on the Hill

http:/ / www.washingtonpost.com/wp-dyn/articles/A61023-2001Oct2.html

Florida Task Force's Recommendation: Give State Police Added Power

http://www.gopbi.com/partners/pbpost/epaper/editions/tuesday/news_4.html

USACM letter regarding encryption controls

http://www.acm.org/usacm/crypto/gregg-crypto-letter.html

pro-Carnivore column employing several jargon techniques

http://writ.news.findlaw.com/commentary/20011001_hodes.html

Net Freedom Fears "Hurt Terror Fight"

http://news.bbc.co.uk/hi/english/uk_politics/newsid_1568000/1568254.stm

Detecting Steganographic Content on the Internet

http:/ / www.citi.umich.edu/techreports/reports/citi-tr-01-11.pdf 
Bill Introduced to Encourage Public-Private Information Sharing http://www.gcn.com/vol1_no1/daily-updates/17197-1.html documents relevant to Homeland Defense http:/ / stinet.dtic.mil/dticrev/vol5-number4.html radio story and background article about new high-tech spy planes (when they fly over Afghanistan, it's a war story; here, it's civil liberties) http:/ / www.npr.org/ramfiles/me/20011002.me.10.ram http://www.fas.org/irp/program/collect/global_hawk.htm Challenges for the Supreme Court in the Wake of Terrorism http:/ / writ.news.findlaw.com/lazarus/20011002.html House Panel Approves Bill Expanding Surveillance http:/ / www.nytimes.com/2001/10/04/national/04RIGH.html the anti-terrorism bill http://www.house.gov/judiciary/hr2975terrorismbill.pdf analysis of the bill http://www.eff.org/Privacy/Surveillance/20011001_house_patriot_analysis.html http://www.epic.org/privacy/terrorism/cong_ltr_10_02_01.html argument against racial profiling in the investigation http://www.law.com/cgi-bin/nwlink.cgi?ACG=ZZZWEVL1BSC Senator Feinstein Urges Major Changes in US Student Visa Program http://www.senate.gov/ feinstein/releases01/stvisas1.htm Democracy in Wartime http:/ / www.nytimes.com/2001/10/03/opinion/03SCHL.html Can the New York Times Count -- or Quote -- Peace Activists? http://www.fair.org/activism/nyt-peace-activists.html A Battle-Ready Net? http://www.businessweek.com/technology/content/oct2001/tc2001101_7845.htm What Went Wrong (with the CIA) 
http://newyorker.com/FACT/

Unscreened Ground Crews Add to Flying Jitters

http://www.latimes.com/news/printedition/california/la-000078980oct03.column

White House, Senate Reach Agreement on Anti-Terrorism Bill

http:/ / www.latimes.com/news/printedition/asection/la-000079336oct04.story

Terror Laws Near Votes in House and Senate

http:/ / www.nytimes.com/2001/10/05/national/05RIGH.html?pagewanted=print Ashcroft Pushes Stronger Antiterrorism Bill

http://www.cnn.com/2001/US/10/04/inv.ashcroft.terrorism/

Toward a Balanced Terrorism Bill

http:/ / www.nytimes.com/2001/10/04/opinion/04THU3.html

Proposed Legislation Significantly Affecting the Computer Profession

http://www.usenix.org/whatsnew/legislation.html

Consortium Responds to More Restrictive Access to Visas by Non-Immigrants http:/ / www.uciep.org/press1.htm

Report Warns of Rights Abuse Risk

http:/ / www.latimes.com/news/printedition/asection/la-000079340oct04.story

Viisage Selected to Deploy the First Face-Recognition System in a US Airport

http:/ / biz.yahoo.com/bw/011004/42302_1.html

Nixed "Holy War" Web Site Offered PGP Encryption Key

http:/ / www.newsbytes.com/news/01/170828.html

Massive Search Reveals No Secret Code in Web Images

http:/ / www.newscientist.com/news/news.jsp?id=ns99991340

Twenty Most Critical Internet Security Vulnerabilities

http:/ /66.129.1.101/top20.htm

Securing the Lines of a Wired Nation

http:/ / www.nytimes.com/2001/10/04/technology/circuits/04SECU.html

Immigrants' Driver's License Bill a Victim of Terrible Timing

http://www.latimes.com/news/printedition/california/la-000079422oct04.column 
Muslim Leaders Condemn Thatcher Attack

http:/ / www.guardian.co.uk/wtccrash/story/0,1300,563247,00.html

"in America, history shows, war does not override the calculus of politics"

http://www.theatlantic.com/unbound/polipro/pp2001-10-03.htm

Bill to Boost Defendants' Rights in Italy Hinders Terrorism War (Berlusconi and his cronies are changing

criminal law to protect themselves)

http://www.latimes.com/news/printedition/asection/la-000079343oct04.story

Senate Favors Federal Airport Screeners

http:/ / www.latimes.com/news/printedition/asection/la-000079338oct04.story

Three Political Websites Downed After Government "Homeland Security" Threat

http:/ / slash.autonomedia.org/article.pl?sid=01/09/30/1859212

Activists and Companies Confront Face Recognition Software

http://www.notbored.org/ciberpais2.html

Carnivore Substitute Keeps Feds Honest

http:/ / www.theregister.co.uk/ content/6/21992.html

New FTC Head Wants "Pause" in Push for Privacy Laws

http://www.computerworld.com/storyba/0,4125,NAV47_STO64453,00.html

http:/ / www.nytimes.com/2001/10/03/technology/03PRIV.html

Zero-Knowledge Shelves Anonymity Tool (that sort of thing probably can't work unless it is built in)

http:/ / news.cnet.com/news/0-1005-200-7412015.html

Seeking Sunlight for a Prisoner in the Chinese Gulag

http:/ / www.latimes.com/news/opinion/commentary/la-000078572oct01.story

argument for a sunset clause in the anti-terrorism bill

http:/ / www.theatlantic.com/politics/nj/taylor2001-10-02.htm

Do New Anti-Terrorism Proposals Pass Constitutional Muster?

http://www.law.com/cgi-bin/nwlink.cgi?ACG=ZZZKRW2IGSC

argument against Bush's proposals to restrict civil liberties 
http:/ / prospect.org/print/V12/18/cole-d.html

The Terrorism Bill Does Too Much and Not Enough

http://www.tnr.com/101501/rosen101501.html

Leahy on "Protecting Constitutional Freedoms in the Face of Terrorism"

http://www.senate.gov/ leahy/press/200110/100301.html

text of the Senate's anti-terrorism bill

http:/ / www.senate.gov/ leahy/press/200110/USA.pdf

http://www.senate.gov/ leahy/press/200110/100401a.html

Indefinite Detention Based Upon Suspicion Under the New Anti-Terrorism Act

http://writ.news.findlaw.com/commentary/20011005_ramasastry.html

Surveillance Warrants Keep Secret Court Busy

http://www.usatoday.com/usatonline/20011004/3508205s.htm

concerns about efforts to revise government controls on strong encryption

http://www.acm.org/usacm/crypto/crypto-controls-memo.html

overly-broad definition of "terrorism" in the Administration's proposal

http://www.acm.org/usacm/terrorist-memo.html

ACLU Calls New Senate Terrorism Bill Significantly Worse

http:/ / www.aclu.org/safeandfree/

"Sunset Clause" Could Trip Up Anti-Terror Bill

http:/ / www.latimes.com/news/printedition/asection/la-000079529oct05.story

Judge Strikes Down Parts of 1996 Terrorism Law

http:/ / www.latimes.com/news/printedition/asection/la-000079527oct05.story

Paul Wolfowitz wants to replace Posse Comitatus (add up their proposals and you're

well on the way to

martial law)

http:/ / www.newsday.com/news/nationworld/nation/wire/sns-ap-attacks-

defense-strategy1004oct04.story

Britain as a Cautionary Tale for a New Age of Surveillance 
http:/ / www.nytimes.com/2001/10/07/ magazine/07SURVEILLANCE.html?pagew anted $=$ all

Florida's Continued Alert Status Criticized

http://www.miami.com/herald/content/news/local/dade/digdocs/112393.htm

recommendations to help secure computing infrastructure against attacks http://www.acm.org/usacm/crypto/comp-sec-memo.html hard-to-evaluate claim that the hijackers used steganography http://abcnews.go.com/sections/primetime/DailyNews/PRIMETIME_011004_steg anography.html

The Supreme Court Returns to a Changed Legal Landscape http:/ / writ.news.findlaw.com/ dorf/20011003.html

Censorship After 9/11: The Bill Maher "Coward" Comment http://www.holtuncensored.com/members/column269.html FBI testimony to the Senate about terrorist groups http://www.fbi.gov/congress/congress01/freeh051001.htm Senator Rethinks Stance on Students http:/ / www.latimes.com/news/printedition/asection/la-000079922oct06.story FAA, Airlines Stalled Major Security Plans http://www.latimes.com/news/nationworld/nation/la-100601air.story Watchdog Sites Shut Down in Interest of National Security http://newsfactor.com/perl/story/13990.html Security Breaches Vary Widely at US Airports http://www.cnn.com/2001/US/10/05/inv.airport.security/ Tighter Airport Security Is Just a Flight of Fancy http:/ / www.latimes.com/news/printedition/california/la-000079574oct05.column criticism of UK's anti-terrorism legislation http://www.magnacartaplus.org/bills/terrorism/index.htm House Votes for More Spy Aid and to Pull in Reins on Inquiry http:/ / www.nytimes.com/2001/10/06/national/06INTE.html 
conservative support for the extreme provisions of the anti-terrorism bill http:/ / www.opinionjournal.com/extra/?id=95001279 http:/ / www.latimes.com/news/printedition/suncommentary/la000080099oct07.story

A High-Tech Home Front (including criticism of face recognition systems) (may not work under Netscape, or Explorer either for that matter) http://www.msnbc.com/news/635417.asp

National ID Cards: One Size Fits All http:/ /www.nytimes.com/2001/10/07/weekinreview/07WAKI.html New Slogan in Washington: Watch What You Say ("a suspension of freedom unlike anything since World War II")

http:/ / www.nytimes.com/2001/10/07/ national/07PRES.html

Demonizing Dissent http:/ / www.workingforchange.com/ article.cfm?ItemID=12064 Now We Really Need Rights http://www.observer.co.uk/comment/story/0,6903,564633,00.html

\section{Jesús Cea Avió}

njcea@hispasec.com

(c) Hispasec, 2001 www.hispasec.com/copyright.asp

Copiado de la lista "noticias@hispasec.com" 09/10/2001 